\title{
Initial Conditions of Inhomogeneous Universe and the Cosmological Constant Problem
}

\author{
Tomonori Totani \\ Department of Astronomy, The University of Tokyo, Hongo, Tokyo 113-0033, Japan \\ E-mail: totani@astron.s.u-tokyo.ac.jp
}

\begin{abstract}
Deriving the Einstein field equations (EFE) with matter fluid from the action principle is not straightforward, because mass conservation must be added as an additional constraint to make rest-frame mass density variable in reaction to metric variation. This can be avoided by introducing a constraint $\delta(\sqrt{-g})=0$ to metric variations $\delta g^{\mu \nu}$, and then the cosmological constant $\Lambda$ emerges as an integration constant. This is a removal of one of the four constraints on initial conditions forced by EFE at the birth of the universe, and it may imply that EFE are unnecessarily restrictive about initial conditions. I then adopt a principle that the theory of gravity should be able to solve time evolution starting from arbitrary inhomogeneous initial conditions about spacetime and matter. The equations of gravitational fields satisfying this principle are obtained, by setting four auxiliary constraints on $\delta g^{\mu \nu}$ to extract six degrees of freedom for gravity. The cost of achieving this is a loss of general covariance, but these equations constitute a consistent theory if they hold in the special coordinate systems that can be uniquely specified with respect to the initial spacelike hypersurface when the universe was born. This theory predicts that gravity is described by EFE with non-zero $\Lambda$ in a homogeneous patch of the universe created by inflation, but $\Lambda$ changes continuously across different patches. Then both the smallness and coincidence problems of the cosmological constant are solved by the anthropic argument. This is just a result of inhomogeneous initial conditions, not requiring any change of the fundamental physical laws in different patches.
\end{abstract}




\section{Contents}

1 Introduction 1

2 Examination of the Action Principle in Relativistic Cosmology 2

3 The Principle of Free Initial Condition 4

4 Gravitational Field Equations for Arbitrary Initial Conditions 5

5 Implications for the Cosmological Constant Problem $\quad 8$

$\begin{array}{llr}6 & \text { Discussion } & 9\end{array}$

\section{Introduction}

The energy density of vacuum appears as the cosmological constant term, $\Lambda$, in the Einstein field equations (EFE). Non-zero $\Lambda$ in the present universe was already implied by some observations in early 1990's [1-5], which were further strengthened by type Ia supernova data $[6,7]$, and finally confirmed by the WMAP data of the cosmic microwave background (CMB) [8]. The energy density inferred from the observed value $\Lambda_{\text {obs }}$ is smaller than the Plank energy density $\left(c^{7} / \hbar G^{2}\right)$ by a factor of $10^{120}$, and smaller than those expected by particle physics theories by at least $10^{60}$, which is the smallness problem of the cosmological constant. It is even more complicated by the coincidence problem that we are living in a very special epoch when the matter density becomes comparable with that of vacuum. In spite of a large number of proposals, there is no compelling candidate of the solution (see [9-12] for recent reviews on theories and observations).

In this work a new theory of gravity, which includes general relativity as a particular case, is proposed to solve the cosmological constant problem, based on reconsideration of the metric degrees of freedom (DOFs) in the action principle to derive the equations of gravitational fields, which are related to constraints on initial conditions. In $\S 2$, we start by an examination of the EFE derivation with fluid matter, pointing out a rather strange aspect that the fluid rest-mass density must be varied at the same time with metric $g_{\mu \nu}$ to obtain the matter energy tensor. This can be avoided by introducing a condition of constant covariant volume element, i.e., $\delta(\sqrt{-g})=0$, in variation of the metric tensor, $\delta g^{\mu \nu}$. Then $\Lambda$ appears in EFE as an integration constant. This is often called the unimodular theory ${ }^{1}$ or equivalently the trace-free EFE, and has been discussed in the literature for a long time, starting from Einstein himself [13-27]. The motivations to introduce this constraint depend on authors: a theory for the internal structure of the electron [13-15], a fundamental atomic length [16], a viewpoint of the little group in the description of massless spin-two particles [17], quantum gravity [19], or the cosmological constant problem [13-15, 20-22, 24-26]. However, to the author's knowledge, the motivation described in this work is not found in previous studies.

\footnotetext{
${ }^{1}$ The unimodular condition is normally defined as $\sqrt{-g}=1$, but in this work we only require the variational condition of $\delta(\sqrt{-g})=0$. It is always possible to make a general coordinate transformation to a system in which $\sqrt{-g}=1$ everywhere [17].
} 
The constant $\sqrt{-g}$ condition does not solve the cosmological constant problem, but gives an important hint. The true physical DOFs for gravity are at most six in the ten components of $g_{\mu \nu}$, after removing four DOFs of coordinate transformation. Correspondingly, four of the ten components of EFE are not dynamical equations, but constraints to initial conditions. This is a result of varying the action with all the ten metric components, while the physical DOFs of gravity is less than ten. This may indicate that EFE are unnecessarily restrictive about initial conditions. The constant $\sqrt{-g}$ condition indeed removes one of these constraints. In $\S 3$, we extend this, and introduce a principle that the gravity theory must be able to solve time evolution for arbitrary initial conditions of inhomogeneous spacetime and matter. In conventional general relativity, only a special universe satisfying the four constraints in EFE is allowed to start. It may be fascinating that a theory of spacetime can predict time evolution starting from any physically possible initial states about spacetime and matter at the birth of the universe. The equations for gravitational fields satisfying this principle will be presented in $\S 4$, by introducing three more constraints on $\delta g^{\mu \nu}$. The cost of getting this is violation of general covariance. However, these equations still give a consistent theory to determine spacetime evolution, if we consider that these equations hold in a uniquely specified coordinate system defined by the initial space-like hypersurface at the birth of the universe. Though the proposed theory loses the beauty of general covariance, it acquires another property that no constraints need to be forced to the initial conditions. Furthermore, as discussed in $\S 5$, the cosmological constant problems are solved simply as a result of inhomogeneous initial conditions and subsequent inflation. General covariance is restored and EFE with a non-zero $\Lambda$ apply in a homogeneous patch of the universe created by inflation.

The sign convention in this work is the same as that of [28], and the fundamental constants $c$ and $G$ will be set equal to unity. Greek indices run from 0 to 3, while Latin indices are for spatial indices running from 1 to 3 . Partial derivatives are represented by colons or $\partial_{\mu}$, while covariant derivatives by semicolons or $\nabla_{\mu}$.

\section{Examination of the Action Principle in Relativistic Cosmology}

EFE can be derived from the principle of least action with the simple Lagrangian density for gravity $\left[L_{G}=-R /(2 \kappa)\right.$, where $R$ is the Ricci scalar and $\left.\kappa=8 \pi\right]$. To derive EFE with perfect fluid, the energy-momentum tensor, $T_{\mu \nu}=(\rho+p) u_{\mu} u_{\nu}-p g_{\mu \nu}$, must be derived by metric variation of the matter Lagrangian $L_{M}$, where $\rho$ and $p$ are energy density and pressure in the fluid rest-frame, respectively, and $u^{\mu}$ is fluid four-velocity. It is possible to find such $L_{M}$, but not quite simple. Here, curl-free fluid of non-relativistic matter $\left(p=0\right.$ and $\left.\rho=\rho_{m}\right)$ is considered for simplicity, where $\rho_{m}$ is the rest mass density in the fluid rest-frame, though it is possible to extend to the case of non-zero pressure and vorticity [29-32]. The fundamental matter Lagrangian is simply given by $L_{M, f}=-\rho_{m}$, but additional conditions of the identity $g_{\mu \nu} u^{\mu} u^{\nu}=1$ and conservation of rest mass $\left[\nabla_{\mu}\left(\rho_{m} u^{\mu}\right)=0\right]$ are necessary with Lagrange multipliers of $\zeta$ and $\eta$, resulting in the following action:

$$
\begin{aligned}
S & =\int\left(L_{G}+L_{M}\right) \sqrt{-g} d^{4} x, \\
L_{M} & =L_{M, f}+\zeta\left(g_{\mu \nu} u^{\mu} u^{\nu}-1\right)+\eta \nabla_{\mu}\left(\rho_{m} u^{\mu}\right) .
\end{aligned}
$$

Variations about $\rho_{m}, u^{\mu}, \zeta$, and $\eta$, but fixing $g_{\mu \nu}$, result in the mass conservation and the Euler equation of motion, $u^{\mu} \nabla_{\mu} u^{\nu}=0$, under appropriate boundary conditions. The Lagrange multipliers are also determined by this process, as $\zeta=-\rho_{m} / 2$ and $\partial_{\mu} \eta=-u_{\mu}$. 
Now consider variation of the action $S$ about metric $g^{\mu \nu}$. To get EFE, the energy momentum tensor must be derived by the standard formula (e.g., [28]):

$$
T_{\mu \nu}=\frac{2}{\sqrt{-g}}\left[\frac{\partial\left(L_{M} \sqrt{-g}\right)}{\partial g^{\mu \nu}}-\frac{\partial}{\partial x^{\lambda}} \frac{\partial\left(L_{M} \sqrt{-g}\right)}{\partial g_{, \lambda}^{\mu \nu}}\right],
$$

and indeed we find $T_{\mu \nu}=\rho_{m} u_{\mu} u_{\nu}$, but only if the same Lagrange multipliers $(\zeta$ and $\eta$ ) determined by the variations about fluid quantities are used. The mass conservation constraint includes the Christoffel symbols $\Gamma_{\rho \sigma}^{\mu}$, and the term coming from variation of $\delta \Gamma_{\mu \lambda}^{\mu}=$ $-\partial_{\lambda}\left(g_{\mu \nu} \delta g^{\mu \nu} / 2\right)$ has a role to cancel the unnecessary term of $\propto \rho_{m} g_{\mu \nu}$ generated by variation of the determinant, $\delta \sqrt{-g}=-\sqrt{-g} g_{\mu \nu} \delta g^{\mu \nu} / 2$. However, it seems rather unbalanced that the Lagrange multipliers are determined solely by fluid dynamics and these must be used also in variation about $g^{\mu \nu}$. If metric is determined by the action principle for fixed fields of $\rho_{m}$ and $u^{\mu}$, the Lagrange multipliers need not be the same as those determined by variation about fluid quantities. In this case the constraints in Lagrangian reduce DOFs of the metric variations $\delta g^{\mu \nu}$, and correspondingly increased DOFs of solutions appear as the freedom of choosing the Lagrange multipliers. Instead, if we require the same multipliers for variations of $g^{\mu \nu}$ and fluid quantities, this is equivalent to requesting that the action is stationary when both the metric and fluid quantities are varied simultaneously satisfying the constraints. In this case all the ten metric components can be varied independently without reduction of DOFs, because the constraints can be met by accordingly changing fluid quantities. This is reasonable for $\zeta$, because variations of $g^{\mu \nu}$ necessarily change the proper time $d s$ and hence four-velocity $u^{\mu}=d x^{\mu} / d s$ for a world line $x^{\mu}(s)$ of a fluid element.

However, the use of the same $\eta$ means that $\rho_{m}$ may also change accordingly with $\delta g^{\mu \nu}$ to satisfy the mass conservation condition. This can be seen more clearly in the FriedmannLemaittre-Robertson-Walker (FLRW) metric in cosmology, where the ii component of EFE is derived from variation about $g^{i i}=-a^{-2}$ keeping $g^{00}=1$, where $a$ is the scale factor. The mass conservation can be written as $\nabla_{\mu}\left(\rho_{m} u^{\mu}\right)=\dot{\rho}_{m}+\Gamma_{\mu 0}^{\mu} \rho_{m}=0$, where $\Gamma_{\mu 0}^{\mu}=3 \dot{a} / a$ and the dot denotes time derivative. Then, if $\rho_{m}(t)$ is fixed, the Hubble parameter $H \equiv \dot{a} / a$ cannot be varied, but in the flat universe case the only physically meaningful quantity about spacetime is $H(t)$. This means that independent variations of $g^{00}$ and $g^{i i}$, which are necessary to derive the full set of EFE in FLRW cosmology, are possible only when $\rho_{m}$ is allowed to vary with $a$ to meet the mass conservation, i.e., $\delta\left(\rho_{m} a^{3}\right)=0$. Alternatively, if we request that $\rho_{m}$ is fixed, there must be a constraint to the relation between $\delta g^{00}$ and $\delta g^{i i}$.

The source of gravity in EFE to determine the spacetime curvature is energy density rather than a total mass in a volume element, and it seems strange if $\rho_{m}$ also needs to change with metric variation. The equivalence principle tells us that gravity force can be erased in a local inertial frame by an appropriate coordinate transformation, and hence the gravity should not affect the physical quantities observed in a local inertial frame including $\rho_{m}$. Therefore I here adopt a principle that rest-frame density of conserving mass (or particle number) should not be altered in the process of finding solutions of gravitational fields by the action principle. This means that variations of $\delta g^{\mu \nu}$ lose one DOF to meet the mass conservation, and we should leave the multiplier $\eta$ as a free field in the solution of gravitational fields. If we split as $\eta \equiv \eta_{f}+\eta_{g}$, where $\eta_{f}$ is that determined by fluid variation (i.e., $\partial_{\mu} \eta_{f}=-u_{\mu}$ ), the 
term including $\eta_{f}$ is absorbed into $T_{\mu \nu}$ and the right-hand-side of EFE is modified as

$$
\begin{aligned}
R_{\mu \nu}-\frac{1}{2} R g_{\mu \nu} & =\kappa T_{\mu \nu}+\Lambda\left(x^{\mu}\right) g_{\mu \nu}, \\
\Lambda\left(x^{\mu}\right) & \equiv \kappa \rho_{m} u^{\lambda} \partial_{\lambda} \eta_{g},
\end{aligned}
$$

where $R_{\mu \nu}$ is the Ricci tensor. Because of the contracted Bianchi identity and the energy conservation of fluid $\left(\nabla_{\mu} T^{\mu \nu}=0\right)$, we find $\partial_{\mu} \Lambda\left(x^{\mu}\right)=0$, i.e., $\Lambda$ must be a constant everywhere in the spacetime. Therefore the change from the original EFE is just adding a cosmological constant, but this is now an integration constant depending on boundary or initial conditions, rather than a fundamental physical constant.

However, the above scheme is still not satisfactory as a general theory to determine the spacetime geometry for fixed matter quantities, because the constraint on metric variation is introduced as the form of mass conservation. The mass conservation is not always satisfied in reality (e.g., fluid composed of decaying particles into radiation). Another constraint depending only on the metric itself but resulting in the same equation would be better, and in fact we can find one. Both the metric variations of $\sqrt{-g}$ and $\nabla_{\mu}\left(\rho_{m} u^{\mu}\right)$ have a form of $\propto g_{\mu \nu} \delta g^{\mu \nu}$, and hence if we introduce the constraint $\delta(\sqrt{-g})=0$ instead of mass conservation, the same result is obtained. The condition of fixed $\sqrt{-g}$ is reasonable if we request that $\rho_{m}$ is fixed against variation of $\delta g^{\mu \nu}$, because $\rho_{m} \sqrt{-g} d^{4} x$ is also a scalar quantity and the only way to keep these two scalars constant is to fix $\sqrt{-g}$. I reached the idea of introducing this constraint purely by the motivation described above. Then I learned, in surveying the literature, that this constraint has been discussed for a long time (see references in Introduction), though motivations were different. In the following it is assumed that this constraint is essential when solutions of gravitational fields are found by the action principle.

\section{The Principle of Free Initial Condition}

The outcome of introducing the constant $\sqrt{-g}$ condition is interesting because $\Lambda$ emerges as an integration constant, but it is not satisfactory for the cosmological constant problem, given that there is no guiding principle to determine its value as an initial condition. However, $\Lambda$ as an integration constant has a different nature from $\Lambda$ being a fundamental physical constant, because it increases one DOF for allowed solutions, and a wider set of initial conditions become possible. In FLRW cosmology with $\Lambda$ as a fundamental physical constant, the Friedmann equation sets a constraint on the allowed initial condition; the Hubble parameter is simply related to energy density at any time in a flat universe. However, if $\Lambda$ is added as an arbitrary integration constant, it can easily be erased by taking a linear combination of the 00 and $i i$ components of EFE, resulting in a second-order time differential equation for the scale factor $a$, allowing any combination of $H=\dot{a} / a$ and $\rho$ as an initial condition. However, this is only for the FLRW metric, and we cannot start with an arbitrary initial condition in an inhomogeneous universe, because $\Lambda$ is a universal constant throughout the spacetime.

In general relativity, the constraints on initial conditions come from the ten components of EFE derived from the action principle with variations of all the ten metric components $\delta g^{\mu \nu}$ independently, though the physical DOFs of gravity are at most six because of the four DOFs of general coordinate transformations. In EFE, the second order time derivatives of the $0 \mu$ metric components, $\partial_{0}^{2} g_{0 \mu}$, do not appear, and the $0 \mu$ components of the Einstein tensor $G_{\mu \nu}=R_{\mu \nu}-R g_{\mu \nu} / 2$ do not include the second-order time derivative of any metric component. Therefore it is natural to regard the spatial components $g_{i j}$ as the dynamical variables, and we 
can always choose a synchronous coordinate system in which $g_{00}=1$ and $g_{0 i}=0$ everywhere for any spacetime geometry. Then the six $i j$ components of EFE determine the evolution of $g_{i j}$, and the additional four $0 \mu$ components of EFE are not dynamical equations but just give constraints on initial conditions. (If they are met at the initial time, the contracted Bianchi identity ensures that they hold at any time.) Therefore time evolution of spacetime cannot be solved for arbitrary $g_{i j}, \partial_{0} g_{i j}$, and $T_{\mu \nu}$ at an initial space-like hypersurface.

Considering physical DOFs of gravitational fields, we may not need to variate the action with all the ten metric components independently. In fact, the constant $\sqrt{-g}$ condition removes one DOF of $\delta g^{\mu \nu}$, and as a result one of the four constraint equations disappears. One may still consider that variation should be done with all the ten metric components, since the action should be stationary not only against $\delta g^{\mu \nu}$ by six DOFs of gravity, but also against those induced by coordinate transformation. However, metric variations generated by infinitesimal coordinate transformation, $x^{\mu} \rightarrow x^{\mu}+\xi^{\mu}$, are $\delta g^{\mu \nu}=\xi^{\mu ; \nu}+\xi^{\nu ; \mu}$. If we consider $\xi^{\mu}$ that is nonzero in an infinitesimal region but zero otherwise, the stationary action condition results in the contracted Bianchi identity and energy conservation after integrating by parts, as written in textbooks (e.g., [28]). These are constraints on the derivatives of EFE rather than EFE themselves. Hence there is no particular reason to require the stationary action against all the ten metric components.

Therefore the standard 10-component EFE may be unnecessarily restrictive as the theory of gravity, and a theory with less constraints on initial conditions may be constructed. We can physically imagine an arbitrary matter distribution embedded in an arbitrary spacetime structure at the initial space-like hypersurface when the universe was born. Though such a universe is not allowed in the standard EFE, it would be fascinating if we have a theory that can predict time evolution for such a universe as well. Here I propose the principle of free initial condition: the gravity theory must be formulated so that time evolution can be solved for arbitrary initial conditions about spacetime and matter. The initial conditions of spacetime should be those for the metric and its time derivative, like many dynamical systems described in physics. The conventional EFE obviously do not satisfy this principle. Introducing the constant $\sqrt{-g}$ condition is not yet satisfactory, because the principle is satisfied only in isotropic and homogeneous FLRW cosmology. Clearly, we need to add three more constraints on $\delta g^{\mu \nu}$ to remove the remaining three constraint equations.

\section{Gravitational Field Equations for Arbitrary Initial Conditions}

It is reasonable to expect that the three more constraints on $\delta g^{\mu \nu}$ are related with the $0 i$ components of EFE, and hence the synchronous coordinate system in which $g_{00}=g^{00}=1$ and $g_{0 i}=g^{i 0}=0$ seems a natural reference frame where the gravity DOFs are easy to treat. Consider time evolution from a space-like hypersurface defined by $x^{0}=t_{i}$. The direction of the normal at each point on this hypersurface is uniquely determined, and $g_{0 i}=g^{0 i}=0$ if we set the spatial coordinate so that $x^{i}$ do not change along this direction. Therefore setting $g_{0 i}=g^{0 i}=0$ is just a matter of coordinate choice, which is unrelated to the physical DOFs of gravity. If we set the constraints of $\delta g^{0 i}=0$ on the metric variations, the $0 i$ components of EFE do not need to hold. However, we cannot set $\delta g^{00}=0$ because it includes a gravity DOF, though it is not independent from $\delta g^{i j}$ because of the constant $\sqrt{-g}$ condition. The six DOFs of gravity are then represented by seven-component variations of $\delta g^{00}$ and $\delta g^{i j}$ with the constraint of $\delta(\sqrt{-g}) \propto g_{00} \delta g^{00}+g_{i j} \delta g^{i j}=0$. Requiring that the action is stationary 
against these variations, we find

$$
R_{\mu \nu}-\frac{1}{2} R g_{\mu \nu}=\kappa T_{\mu \nu}+\Lambda_{0} g_{\mu \nu}+\Xi_{\mu \nu},
$$

where

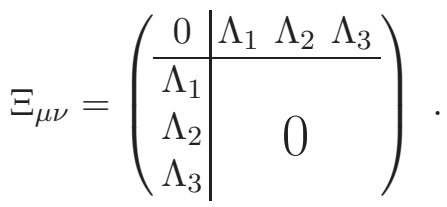

Here, $\Lambda_{0}\left(x^{\mu}\right)$ is a Lagrange multiplier field corresponding to the constant $\sqrt{-g}$ condition, and $\Lambda_{i}\left(x^{\mu}\right)$ are arbitrary fields to make the $0 i$ components of EFE ineffective. Of course, the set of $\left(\Lambda_{0}, \Lambda_{i}\right)$ is not a vector, but $\Lambda_{0}$ is a scalar and $\Lambda_{i}$ are components ${ }^{2}$ of $\Xi_{\mu \nu}$. The contravariant version $\Xi^{\mu \nu}$ also satisfies $\Xi^{00}=\Xi^{i j}=0$, and their nonzero components are denoted as $\Xi^{0 i}=\Xi^{i 0}=\Lambda^{i}=g^{00} g^{i j} \Lambda_{j}$.

However, a problem of this equation as a theory for gravitational fields is that it violates general covariance, because the above condition $\delta g^{0 i}=0$ is dependent on the choice of coordinate systems, in contrast to the constant $\sqrt{-g}$ condition. Nevertheless, general covariance is not necessarily indispensable for a theory to determine spacetime evolution. If we can specify a unique coordinate system for a given spacetime, equations that hold only in such a system can be a consistent theory. Here we take this option. However, the synchronous condition is not sufficient to specify such a system, because it does not uniquely specify the coordinate system, the Lorentz transformation being an example. The form of $\Xi_{\mu \nu}$ does not keep eq. (4.2) by a Lorentz transformation, and hence eqs. (4.1) with eq. (4.2) cannot be a general form for any synchronous coordinate systems.

To avoid this problem, it is assumed that any spacetime realized in nature has a finite space-like physical boundary into the past as an initial condition. We do not know how a spacetime is born, but at least the only one example that we know, i.e., our universe, seems to satisfy this. If we can define a physically unique coordinate with respect to this initial spacelike hypersurface, and if equations hold only within it, they become a consistent theory to predict time evolution of spacetime starting from the initial hypersurface, even if they violate general covariance. We define a synchronized time $x^{0}=t_{s}$ on the initial hypersurface, and the spacetime does not extend to the region of $x^{0}<t_{s}$. If we set a spatial coordinate system at $x^{0}=t_{s}$, the synchronous coordinate system starting from this is uniquely determined throughout this spacetime. Only transformations within spatial coordinates $\left[x^{i}=f^{i}\left(x^{j}\right)\right]$ are allowed to keep the synchronous condition at any point in the spacetime and $x^{0}=t_{s}$ on the initial hypersurface. In such a set of coordinate systems, the form of $\Xi_{\mu \nu}$ in eq. (4.2) is unchanged. [It is also kept against a transformation including only time coordinate, $\left.x^{\prime 0}=f^{0}\left(x^{0}\right)\right]$. Therefore, if eqs. (4.1) and (4.2) are assumed to hold only in these coordinate systems, they give a consistent theory to predict time evolution.

Then the principle of free initial conditions is now satisfied. The evolution of gravitational fields $g_{i j}$ is determined by eq. (4.1) with the form of $\Xi_{\mu \nu}$ given in eq. (4.2). The seven equations of the 00 and $i j$ components include $\Lambda_{0}\left(x^{\mu}\right)$, and this can be erased resulting in six second-order time differential equations for $g_{i j}$. Then we can take any combination of $g_{i j}$, their time derivatives, and $T_{\mu \nu}$ as an initial condition. The initial values of $\Lambda_{0}$ and

\footnotetext{
${ }^{2}$ The character $\Xi$ has been chosen because $\Xi$ has three non-zero components and it looks similar to the Chinese character "three".
} 
$\Lambda_{i}$ are also determined by the initial conditions of spacetime and matter. In EFE, energy conservation of matter is automatically satisfied by the contracted Bianchi identity, but here the least action condition of matter must be independently required to ensure $\nabla_{\mu} T^{\mu \nu}=0$. Then four-divergence of eq. (4.1) gives four first-order time differential equations to determine evolution of $\Lambda_{0}$ and $\Lambda^{i}$ as

$$
\nabla_{\mu}\left(\Lambda_{0} g^{\mu \nu}+\Xi^{\mu \nu}\right)=0
$$

Obviously this includes the standard general relativity $\left(\Lambda_{0}=\Lambda_{i}=0\right)$, and hence this is an extension of general relativity.

Though this theory violates general covariance, eqs. (4.1) is written in a generally covariant form, and hence these equations can be extended to any coordinate systems in the same form, if we define the ordinary tensor transformation law for $\Xi_{\mu \nu}$. It violates general covariance in the sense that the expression of $\Xi_{\mu \nu}$ takes a special form of eq. (4.2) in a special set of coordinate systems. Violation of general covariance for a theory of gravity may not be unreasonable if we consider the following points. It is standard to start with the Lagrangian density of gravity being the Ricci scalar, $L_{G}=-R /(2 \kappa)$, but a peculiar aspect is that $R$ includes the second-order time derivatives of $g_{\mu \nu}$, while normally Lagrangians include up to first-order derivatives of dynamical variables. It is possible to derive EFE starting from a Lagrangian that does not include second-order time derivatives [28], by defining

$$
Q \equiv g^{\rho \sigma}\left(\Gamma_{\rho \nu}^{\mu} \Gamma_{\sigma \mu}^{\nu}-\Gamma_{\rho \sigma}^{\nu} \Gamma_{\nu \mu}^{\mu}\right)
$$

which is related to $R$ as

$$
R \sqrt{-g}=Q \sqrt{-g}+\frac{\partial}{\partial x^{\mu}}\left[W^{\mu} \sqrt{-g}\right]
$$

where

$$
W^{\mu} \equiv g^{\rho \sigma} \Gamma_{\rho \sigma}^{\mu}-g^{\mu \rho} \Gamma_{\rho \sigma}^{\sigma} .
$$

Therefore the action principle gives the same result if we take $L_{G}=-Q /(2 \kappa)$ and variations of $W^{\mu}$ are fixed to zero at integration boundaries. Another good point of this Lagrangian is the quantities that should be fixed at boundaries. In the case of the conventional Lagrangian, variations of $\Gamma_{\rho \sigma}^{\mu}$ that include time derivatives of $g_{\mu \nu}$ must be fixed to zero at boundaries, because

$$
\delta[R \sqrt{-g}]=\delta\left(g^{\mu \nu} R_{\mu \nu} \sqrt{-g}\right)=G_{\mu \nu} \delta g^{\mu \nu} \sqrt{-g}+\frac{\partial}{\partial x^{\mu}}\left(w^{\mu} \sqrt{-g}\right),
$$

where

$$
w^{\mu}=g^{\rho \sigma} \delta \Gamma_{\rho \sigma}^{\mu}-g^{\mu \rho} \delta \Gamma_{\rho \sigma}^{\sigma} .
$$

However, in ordinary steps to derive the Euler-Lagrange equation of motion from the action principle, first-order time derivatives of dynamical quantities (in this case $\Gamma_{\rho \sigma}^{\mu}$ ) need not be fixed at boundaries. If we take $L_{G} \propto Q$, indeed we need to fix only $\delta g^{\mu \nu}$ to zero at boundaries, because

$$
\delta[Q \sqrt{-g}]=G_{\mu \nu} \delta g^{\mu \nu} \sqrt{-g}-\frac{\partial}{\partial x^{\mu}}\left[\left(\delta g^{\rho \sigma} \Gamma_{\rho \sigma}^{\mu}-\delta g^{\mu \rho} \Gamma_{\rho \sigma}^{\sigma}\right) \sqrt{-g}+W^{\mu} \delta(\sqrt{-g})\right]
$$


and the four-divergence term includes only $\delta g^{\mu \nu}$ without $\delta \Gamma_{\rho \sigma}^{\mu}$. Then $Q$ seems a more natural Lagrangian density than $R$ to derive the Einstein tensor $G_{\mu \nu}$, but $Q$ is not a scalar, and the action becomes dependent on the global properties of a specified coordinate system. Lagrangian density should be closely related to the energy density, and $Q$ has a similar form (quadratic in $\Gamma_{\rho \sigma}^{\mu}$ ) to the energy-momentum psudotensor of gravitational fields, which is not a tensor. These considerations imply that gravity is essentially global and dependent on coordinate systems, and it may not be surprising that the constraints on $\delta g^{\mu \nu}$ to extract six physical DOFs of gravity are expressed in a simple form in special coordinate systems determined by the initial space-like hypersurface.

\section{Implications for the Cosmological Constant Problem}

The extended theory of gravity proposed here allows the cosmological "constant" $\Lambda_{0}$ to change on the initial space-like hypersurface. In accordance with the standard paradigm of the inflationary universe [33-38], here it is assumed that the universe started with a highly inhomogeneous condition. Then $\Lambda_{0} g_{\mu \nu}$ and $\Xi_{\mu \nu}$ would have fluctuations with amplitudes similar to that of matter energy tensor $\kappa T_{\mu \nu}$ at that time. To realize an isotropic and homogeneous universe as observed today, inflation must occur at least in some regions in the whole universe. Let $\Lambda_{\phi}=\kappa \rho_{\phi}$ be the cosmological constant corresponding to the vacuum energy density $\rho_{\phi}$ of the inflaton field $\phi$. Quantitative conditions for successful inflation must be investigated numerically, but we expect that there are some regions where $\Lambda_{\phi} g_{\mu \nu}$ is dominant compared with $\Lambda_{0} g_{\mu \nu}+\Xi_{\mu \nu}$ in eq. (4.1). Then such regions would start inflation, and $\Lambda_{0}$ and $\Lambda^{i}$ evolve by eqs. (4.3). Intuitively, $\Lambda^{i}$ should become zero asymptotically, and consequently $\Lambda_{0}$ becomes a constant by eqs. (4.3), because the universe becomes isotropic and homogeneous by exponential expansion.

This can be examined more quantitatively if the background metric is nearly isotropic. We can treat $\Lambda_{0}$ and $\Lambda^{i}$ perturbatively in the flat FLRW metric, and eqs. (4.3) become

$$
\begin{aligned}
\partial_{0} \Lambda_{0}+\partial_{i} \Lambda^{i} & =0 \\
-\frac{\partial_{i} \Lambda_{0}}{a^{2}}+\partial_{0} \Lambda^{i}+5 H \Lambda^{i} & =0 .
\end{aligned}
$$

From these two equations we can derive the equation for $\Lambda_{0}$ as

$$
\ddot{\Lambda}_{0}+5 H \dot{\Lambda}_{0}+\frac{\Delta \Lambda_{0}}{a^{2}}=0,
$$

where $\Delta$ is the Laplacian in the comoving coordinate. The characteristic inhomogeneity scale of $\Lambda_{0}$ would be the horizon scale $H^{-1}$ just before inflation, and consider a Fourier mode of this scale with a comoving wavenumber $\left|\vec{k}_{i c}\right| \sim H$, i.e., $\Delta \Lambda_{0}=-k_{i c}^{2} \Lambda_{0}$, where we define $a=1$ at the beginning of inflation. Once the inflation starts at $t=t_{s i}$, the evolution is $a=\exp \left[H\left(t-t_{s i}\right)\right]$ with a nearly constant $H$, and this scale should soon become superhorizon. Though $\left|\Lambda_{0}\right|$ is accelerated to a larger value by the force $k_{i c}^{2} \Lambda_{0} / a^{2}$, the friction force $-5 H \dot{\Lambda}_{0}$ limits the rate of $\Lambda_{0}$ evolution as $\dot{\Lambda}_{0} / \Lambda_{0} \lesssim\left(k_{i c}^{2} / 5 a^{2} H\right) \sim H a^{-2}$, and hence $\Lambda_{0}$ would become a constant. Eq. (5.2) indicates that $\Lambda^{i}$ should decay as $\propto \exp (-5 H t)$ when $5 H\left|\Lambda^{i}\right| \gtrsim k_{i c}\left|\Lambda_{0}\right| / a^{2}$, and hence $a\left|\Lambda^{i}\right| /\left|\Lambda_{0}\right|$ is limited to be $\lesssim k_{i c} /(5 a H) \sim a^{-1}$. This means that $\Xi^{\mu \nu}$ is negligible compared with $\Lambda_{0} g^{\mu \nu}$ after inflation. Then finally $\Lambda_{0} g^{\mu \nu}+\Xi^{\mu \nu}$ would nearly become a cosmological constant term $\Lambda_{0, f} g^{\mu \nu}$ in a homogeneous patch of the universe created by inflation, where $\Lambda_{0, f}$ is a universal constant within the patch, but its value can 
be positive or negative and changes continuously on the comoving scale of $k_{i c}$. At the end of the inflation, the contribution $\Lambda_{\phi}$ disappears, and the final effective cosmological constant in a patch is $\Lambda_{f} \equiv \Lambda_{0, f}+\Lambda_{\text {vac }}$, where $\Lambda_{\text {vac }}\left(=\kappa \rho_{\text {vac }} \ll \Lambda_{\phi}\right)$ is the microscopic vacuum energy density in the universe today from any contributing sources including quantum zero-point fluctuations.

The value of $\Lambda_{\mathrm{vac}}$ is expected to be much larger than $\Lambda_{\text {obs }}$ (i.e., the smallness problem), but the amplitude of $\Lambda_{0, f}$ fluctuation would be even larger. Then there should be regions where $\left|\Lambda_{f}\right| \lesssim \Lambda_{\text {obs }}$ in a successfully inflated portion of the universe. This is the region that is habitable for an intelligent life, because patches with $\Lambda_{f} \ll-\Lambda_{\text {obs }}$ should have collapsed much earlier than the present epoch, and formation of structure and galaxies does not occur in patches with $\Lambda_{f} \gg \Lambda_{\text {obs }}$ because of too fast expansion. Therefore the smallness problem is solved by the anthropic argument [39]. The comoving width of the regions of $\left|\Lambda_{f}\right| \lesssim \Lambda_{\text {obs }}$ is much smaller than the initial inhomogeneity scale $k_{i c}^{-1}$ by a factor of $\sim \Lambda_{\text {obs }} / \Lambda_{0, f}$, and the total number of $e$-foldings from the beginning to the end of inflation must be sufficiently large to make this width much larger than the present-day Hubble horizon. The fractional change $\delta \Lambda_{f} / \Lambda_{f}$ is of order unity within the regions of $\left|\Lambda_{f}\right| \lesssim \Lambda_{\text {obs }}$, but those of $\Lambda_{0, f}$ and any other physical quantities (e.g., matter density, properties of inflation, and density fluctuation amplitude) are negligibly small.

It is expected that $\Lambda_{f}$ changes linearly with a spatial position within the regions of $\left|\Lambda_{f}\right| \lesssim \Lambda_{\text {obs }}$, because $\Lambda_{\text {obs }}$ is much smaller than the typical fluctuation amplitude of $\Lambda_{0, f}$. Then the prior probability distribution function $P_{\mathrm{pri}}\left(\Lambda_{f}\right)$ should be almost constant per unit $\Lambda_{f}$. The probability distribution to observe $\Lambda_{f}$ should be $P_{\text {pri }}$ multiplied by the efficiency of creating intelligent life, $\epsilon_{\text {life }}\left(\Lambda_{f}\right)$. It has been shown that, under the assumption of constant $P_{\text {pri }}$ and estimating $\epsilon_{\text {life }}\left(\Lambda_{f}\right)$ by galaxy formation efficiency, the probability for us to observe $\Lambda_{f} \sim \Lambda_{\text {obs }}$ is not extremely small [40,41]. Moreover, the probability of finding $\left|\Lambda_{f}\right| \ll \Lambda_{\text {obs }}$ is small because $P_{\text {pri }}\left(<\left|\Lambda_{f}\right|\right) \propto\left|\Lambda_{f}\right|$, and hence the coincidence problem is also solved.

This picture is analogous to the concentration of human population to coastal areas on Earth, if we regard $\Lambda_{f}$ as altitude above the sea level; we cannot live under the sea level, while it is hard to live in high positive altitude regions. Therefore I call this the coastal universe hypothesis.

\section{Discussion}

The coastal universe scenario predicts that the present-day cosmological constant $\Lambda_{f}$ should vary at different positions, on the comoving scale of initial inhomogeneity before inflation. However, if inflation is sufficient, the expected change within the Hubble horizon should be negligible, and therefore a more practical prediction is that the observed universe should be described exactly by the standard $\Lambda \mathrm{CDM}$ model. The high precision experimental tests on general relativity on the solar system scales are not affected. The current small $\Lambda_{\text {obs }}$ is a result of huge cancellation between $\Lambda_{0, f}$ and $\Lambda_{\mathrm{vac}}$, and the latter should gravitate. This may be tested experimentally, e.g., by examination of gravitational properties of the Casimir energy [11]. Since the proposed theory changes DOFs of gravitational fields, implications for quantum gravity theory would be interesting. Gravitational wave background radiation would be generated during inflation by quantum fluctuation of metric, and the prediction by the proposed theory may be different from the standard one because of the DOFs of $\Lambda_{0} g_{\mu \nu}+\Xi_{\mu \nu}$, though this becomes a cosmological constant by inflation at the classical level. Examination 
of such prediction is beyond the scope of this work, but this may be tested by the $B$-mode polarization of $\mathrm{CMB}$ or direct detection experiments in future.

Though the coastal universe hypothesis uses the anthropic argument, the change of $\Lambda_{f}$ across different homogeneous patches of the universe is just a result of inhomogeneous initial conditions under the same fundamental physical laws. Physical quantities other than $\Lambda_{f}$ do not change, and $\Lambda_{f}$ should have a constant prior probability distribution $P_{\text {pri }}$ per unit $\Lambda_{f}$. This is in contrast to some other explanations of $\Lambda_{\text {obs }}$ based on the anthropic argument (e.g., string landscape [42]), in which not only $\Lambda$ but also other physical quantities (like density fluctuation amplitude) and even the fundamental physical laws or constants may also change. The anthropic argument may not simply work in these cases [43-45]. It is also highly uncertain whether a constant $P_{\text {pri }}\left(\Lambda_{f}\right)$ is realized in some other anthropic scenarios [46]. Astronomy is still showing a remarkable development by large projects in wide wavelength ranges, and our understanding of galaxy formation, star and planet formation, and even the origin of life will be further improved in the future. Then we may be able to calculate the

observational probability distribution of $\Lambda_{f}$ with a more realistic estimate of $\epsilon_{\text {life }}\left(\Lambda_{f}\right)$, giving a more quantitative test of the coastal universe scenario that varies only $\Lambda_{f}$ with a flat prior probability distribution.

\section{References}

[1] G. Efstathiou, W. J. Sutherland and S. J. Maddox, Nature 348, 705 (1990).

[2] M. Fukugita, K. Yamashita, F. Takahara, and Y. Yoshii, Astrophys. J. 361, L1 (1990)

[3] Y. Yoshii, Astrophys. J. 403, 552 (1993)

[4] L. M. Krauss and M. S. Turner, Gen. Rel. Grav. 27, 1137 (1995)

[5] J. P. Ostriker and P. J. Steinhardt, Nature 377, 600 (1995).

[6] A. G. Riess et al. [Supernova Search Team Collaboration], Astron. J. 116, 1009 (1998)

[7] S. Perlmutter et al. [Supernova Cosmology Project Collaboration], Astrophys. J. 517, 565 (1999)

[8] D. N. Spergel et al. [WMAP Collaboration], Astrophys. J. Suppl. 148, 175 (2003)

[9] J. Frieman, M. Turner and D. Huterer, Ann. Rev. Astron. Astrophys. 46, 385 (2008) [arXiv:0803.0982 [astro-ph]].

[10] R. R. Caldwell and M. Kamionkowski, Ann. Rev. Nucl. Part. Sci. 59, 397 (2009) [arXiv:0903.0866 [astro-ph.CO]].

[11] J. Martin, Comptes Rendus Physique 13, 566 (2012) [arXiv:1205.3365 [astro-ph.CO]].

[12] D. H. Weinberg, M. J. Mortonson, D. J. Eisenstein, C. Hirata, A. G. Riess and E. Rozo, Phys. Rept. 530, 87 (2013)

[13] A. Einstein, Sitzungsber. Preuss. Akad. Wiss. Berlin (Math. Phys.) 349-56 (1919)

[14] A. Einstein, The Principle of Relativity (London: Methuen) (1923) (Engl. transl.)

[15] A. Einstein, The Principle of Relativity (Mineola, NY: Dover) (Lorentz, H.A. et al., ed.), reprinted (1952), paper X.

[16] J. L. Anderson and D. Finkelstein, Am. J. Phys. 39, 901 (1971).

[17] J. J. van der Bij, H. van Dam and Y. J. Ng, Physica 116A, 307 (1982).

[18] W. Buchmuller and N. Dragon, Phys. Lett. B 207, 292 (1988). 
[19] W. G. Unruh, Phys. Rev. D 40, 1048 (1989).

[20] M. Henneaux and C. Teitelboim, Phys. Lett. B 222, 195 (1989).

[21] Y. J. Ng and H. van Dam, Phys. Rev. Lett. 65, 1972 (1990).

[22] Y. J. Ng and H. van Dam, J. Math. Phys. 32, 1337 (1991).

[23] D. R. Finkelstein, A. A. Galiautdinov and J. E. Baugh, J. Math. Phys. 42, 340 (2001) [gr-qc/0009099].

[24] Y. J. Ng and H. van Dam, Int. J. Mod. Phys. D 10, 49 (2001) [hep-th/9911102].

[25] L. Smolin, Phys. Rev. D 80, 084003 (2009) [arXiv:0904.4841 [hep-th]].

[26] G. F. R. Ellis, H. van Elst, J. Murugan and J. P. Uzan, Class. Quant. Grav. 28, 225007 (2011).

[27] G. F. R. Ellis, Gen. Rel. Grav. 46, 1619 (2014) [arXiv:1306.3021 [gr-qc]].

[28] L.D. Landau and E.M. Lifshitz, "The Classical Theory of Fields", Elsevier (1975).

[29] B. F. Schutz, Phys. Rev. D 2, 2762 (1970).

[30] J. D. Brown, Class. Quant. Grav. 10, 1579 (1993) [gr-qc/9304026].

[31] N. J. Poplawski, Phys. Lett. A 373, 2620 (2009) [arXiv:0806.2283 [gr-qc]].

[32] H. Fukagawa and Y. Fujitani, Prog. Theo. Phys. 124, 517 (2010)

[33] A. A. Starobinsky, JETP Lett. 30, 682 (1979) [Pisma Zh. Eksp. Teor. Fiz. 30, 719 (1979)].

[34] D. Kazanas, Astrophys. J. 241, L59 (1980).

[35] A. H. Guth, Phys. Rev. D 23, 347 (1981).

[36] K. Sato, Mon. Not. Roy. Astron. Soc. 195, 467 (1981).

[37] A. D. Linde, Phys. Lett. B 108, 389 (1982).

[38] A. Albrecht and P. J. Steinhardt, Phys. Rev. Lett. 48, 1220 (1982).

[39] S. Weinberg, Phys. Rev. Lett. 59, 2607 (1987).

[40] G. Efstathiou, Mon. Not. Roy. Astron. Soc. 274, L73 (1995).

[41] H. Martel, P. R. Shapiro and S. Weinberg, Astrophys. J. 492, 29 (1998).

[42] L. Susskind, in Carr, B. (ed.): "Universe or multiverse?", Cambridge (2009) [hep-th/0302219].

[43] M. Tegmark and M. J. Rees, Astrophys. J. 499, 526 (1998).

[44] A. Aguirre, Phys. Rev. D 64, 083508 (2001).

[45] M. L. Graesser, S. D. H. Hsu, A. Jenkins and M. B. Wise, Phys. Lett. B 600, 15 (2004).

[46] J. Garriga and A. Vilenkin, Phys. Rev. D 61, 083502 (2000). 